MAX-PLANCK-INSTITUT FÜR WISSENSCHAFTSGESCHICHTE

Max Planck Institute for the History of Science

2015

PREPRINT 473

Christoph Lehner, Helge Wendt

Mechanik in der Querelle des Anciens

et des Modernes 



\title{
Mechanik in der Querelle des Anciens et des Modernes
}

\author{
Christoph Lehner, Helge Wendt, MPIWG
}

Der Aufsatz wird erscheinen im Tagungsband Allelopoiese - Konzepte zur Beschreibung kulturellen Wandels, herausgegeben von Prof. Dr. Johannes Helmrath, Dr. Eva Marlene Hausteiner und Dr. Ulf Jensen, De Gruyter, Berlin.

Since the Ancients (according to Pappus) considered mechanics to be of the greatest importance in the investigation of nature and science and since the moderns-rejecting substantial forms and occult qualities-have undertaken to reduce the phenomena of nature to mathematical laws, it has seemed best in this treatise to concentrate on mathematics as it relates to natural philosophy. ${ }^{1}$

Dies ist der erste Satz des Vorworts, den Issac Newton 1687 seinem Hauptwerk den Philosophiae Naturalis Principia Mathematica voranstellte. Es mag überraschen, dass die Revolution in Mathematik und Physik mit einer Anleihe an den antiken Mathematiker Pappos von Alexandria begann. Newtons Beiträge zur reinen und angewandten Mathematik, in der Optik und Chemie, und insbesondere seine Mechanik und Kosmologie wurden bereits zu Newtons Zeiten als eine Revolution wahrgenommen. ${ }^{2}$ Im Gegensatz zu der durch die Revolutions-Metapher hervorgerufenen Erwartung einer allumfassenden Neuheit, die Gegenstand, Methode und Darstellungsweise umfasste, betont Newton in den Principia gleich zwei Kontinuitäten mit antiken Vorläufern: Erstens definiert Newton sein Werk mit dem vorangestellten Pappos-Bezug als eine Fortschreibung antiker Mathematik. Und zweitens gibt Newton, seine durchaus bahnbrechenden physikalischen Erkenntnisse in einer, von ihm (und vielen anderen) als antik definierten und idealisierten Formsprache wieder. Um diesen Bezug auf die Antike zu verstehen, soll im Folgenden diskutiert werden, welche Rolle Newtons Wahrnehmung und Bewertung der antiken Wissenschaft für sein Projekt einer neuen rationalen Mechanik und für den Formalismus spielten, den er in den Principia verwendete. Für das Thema dieses Bandes ist insbesondere interessant, dass diese Berufung auf die Antike eine allelopoietische Konstruktion impliziert, die sich grundsätzlich von dem modernen Bild der antiken Wissenschaft unterscheidet.

\footnotetext{
${ }^{1}$ Newton, Principia. Author's Preface to the Reader, 381.

${ }^{2}$ Dies betont z.B. Cohen (1980, 3-38) in seinem Standardwerk zur Rezeptionsgeschichte Newtons.
} 
Der Kontext für Newtons Verknüpfung seines Werks mit der Antike ist die Querelle des anciens et modernes, die seit der Mitte des 17. Jahrhunderts ein zentraler Topos intellektueller Debatten war. Der weit ins Folgejahrhundert hineinreichende Streit über die kulturelle Überlegenheit der Antike oder der Moderne, ist in der heutigen Wahrnehmung vornehmlich ein Thema der Literaturgeschichte über eine Notwendigkeit - oder eben nicht - einer Neuausrichtung und Neuerfindung von literarischen Narrationsmustern. ${ }^{3}$ Diesen "Kampf" zwischen unterschiedlichen Lagern, wie denn die Beziehungen zwischen zwei Epochen zu bewerten seien, lässt sich nicht allein auf zwei Parteien beschränken, die sich einteilen ließen in eine, der Antike positiv gegenüber stehende, und eine zweite, die Antike ablehnende. ${ }^{4}$ Dietrich Hardt hat den seit der Renaissance andauernden Streit als eine Vielzahl von "Konstruktionsmechanismen" beschrieben, die sich an zwei Gegenpolen orientierten:

Ich möchte im folgenden an einigen historischen Beispielen zeigen, welche Bilder der Antike und welche Konstruktionsmechanismen im Untersuchungszeitraum zwischen etwa 1650 und 1810 aus diesen Kämpfen hervorgegangen sind, wie sie schließlich das Alte in jene Alterität transformierten, an der die Epoche der Moderne einerseits ihren eigenen, dauernd fortschreitenden und permanent sich verändernden offenen Prozeßcharakter, andererseits aber so etwas wie die Orientierungspunkte in einem Fluß permanenten Wandels ablesen konnte. ${ }^{5}$

Unwesentlich verschieden dazu, hatte schon 1859 Hippolyte Rigault die Querelle des anciens et des modernes definiert, da die beiden Grundpositionen doch einem menschlichen Grundbedürfnis sowohl nach Tradition wie nach Fortschritt entsprächen. ${ }^{6}$

Der Streit ist für die Herausbildung von kritischen Methoden in der Literaturwissenschaft grundlegend, jedoch bezieht er eben auch die sich herausbildenden mathematischen Methoden in der Naturphilosophie ein. ${ }^{7}$ Hier war umstritten, inwiefern antike Methoden und Argumentationsformen für die moderne Wissenschaft verbindlich seien. Zugleich beinhaltet die Debatte auch radikal verschiedene Vorstellungen von der Reichweite und Form antiker Wissenschaft.

\footnotetext{
${ }^{3}$ Vgl. Warnick (1982).

${ }^{4}$ Levine (1981).

${ }^{5}$ Hardt (1994), 92.

${ }^{6}$ Rigault (1859), 1.

${ }^{7}$ Vgl. u.a. Tadié (2013). 
Newtons Konstruktion, aus dem eingangs angeführten Zitat, einer inhaltlich und formal überlegenen antiken Wissenschaft, die auch für moderne Bemühungen einen verbindlichen Rahmen bildet, steht dabei in scharfem Kontrast mit dem Geschichtsbild seiner Rezipienten, der mathematischen Physiker des 18. Jahrhunderts auf dem europäischen Kontinent, wie wir im abschließenden Teil des Artikels sehen werden. Um den historischen Kontext dieses Bruches zwischen Newtons Idealen und dessen Rezeption zu verstehen, muss genau dieser Zusammenhang der Kontroverse der Alten und der Modernen um 1700 beleuchtet werden. In den Jahrhunderten zuvor wurden bereits ähnliche Fragen um die Gültigkeit antiker Lehren, in Teilen auch kontrovers behandelt, jedoch weniger vor einem Hintergrund eines historischen Bruches zwischen Antike und Moderne, der besonders in der Querelle des anciens et modernes thematisiert wurde.

Zwei Orte, zum einen Paris und zum anderen London, sind die Hauptschauplätze dieser Auseinandersetzung. An der Pariser Académie entwickelte sich ab der Mitte des 17. Jahrhunderts eine lang andauernden Debatte darüber, ob die literarischen Sujets und Formen antiker Autoren nicht denen moderner Autoren überlegen seien. Diese besonders als Kritik an Konkurrenten im Zirkus der Dichtung und der Literaturkritik gemeinte Überhöhung antiker Dichtkunst entwickelte verschiedene Gegenströmungen. Eine von ihnen setzte eine ,gallische Antike" ${ }^{\Perp 8}$ der griechisch-römischen entgegen, womit es zu einer Pluralisierung der Antike kam und die Verflechtung beider Antiken zu einem bis weit in 18. Jahrhundert hineinreichenden eigenen Streitpunkt avancierte. ${ }^{9}$ Eine andere Strömung bemühte sich um die Rettung von als neu, modern und fortschrittlich - vielleicht eben auch als überlegen - bewerteten literarischen Formen und Themen. Auf lange Sicht gab die Querelle den Blick frei auf das, was letztendlich beide Seiten von der jeweils anderen einforderten: nämlich eine historische Kontextualisierung der Gegenstände, für die sie Partei ergriffen.

In diesem historistischen Sinne entstand ein neuer Streitherd, diesmal jenseits des Kanals, in England. Diese zweite Querelle war eine gezielte Provokation, die zuerst von William Temple in seinem Essay upon ancient and modern learning 1690 lanciert wurde. Diese entstand in einem eher lokal zu nennenden Zusammenhang von intellektuellem Wettstreit und wissenschaftlichen Kontroversen. Sie bezieht sich darüber hinaus auf französische Autoren, Texte und Kontroversen, und findet gleichzeitig eigenständige Antworten und

\footnotetext{
${ }^{8}$ Vgl. zu den Konjunkturen dieser Geschichtsinterpretation. Duval (1989), 177-185.

${ }^{9}$ Nicolet (2001), 1627-1637.
} 
Themenfelder. ${ }^{10}$ Dabei legte Temple sein Verständnis von der Bedeutung der Antike für die Moderne anders an, als dies die meisten französischen Autoren getan hatten. Er plädierte weniger für ein Studium der antiken Literatur als Vorlage für eine moderne Literatur, sondern stellte fest, dass die antiken Werke einen ganz autonomen Wert besaßen. Zudem erweiterte Temple das Feld der Querelle - so wie dies auch vorher schon in Frankreich geschehen war und schloss die Leistungen antiker Autoren auf dem Feld der Naturphilosophie und Mathematik in seine Betrachtungen ein. Temple argumentierte, dass das Wissen der Alten in einer „république de lettres“ der Antike entstanden sei, die sich nicht allein auf einen abgeschlossenen griechisch-römischen Kulturraum beschränkte, sondern ihrerseits auf ägyptischen, phönizischen, kretischen und babylonisch-chaldäischen Vorläufern aufsaß. Das Wissen der Alten, so Temple in pythagoreischem Duktus sei ,transmigriert“ in die moderne Zeit und forderte nun die Philosophen und Literaten heraus, die Ende des 17. Jahrhunderts die Neuheit ihres Schaffens unterstrichen, wie Descartes oder Hobbes. ${ }^{11}$ Beiden räumte Temple durchaus ihr Recht auf besonderes Erklärungspotential der aktuellen Lage der Menschheit ein. Weder konnten sich beide Autoren von den Alten losmachen, denn zu häufig bezogen sie sich auf diese, zitierten, paraphrasierten und verwendeten deren Kategorien und Begriffssysteme. Noch waren Aristoteles, Plato oder Epikur für die Ausbildung in den verschiedenen Wissenschaftsbereichen - und hier findet sich der im Titel deutliche pädagogische Anspruch der Schrift - durch zeitgenössische wissenschaftliche und literarische Werke obsolet geworden (Temple 1690, 468).

1704 veröffentlichte Temples' Sekretär Jonathan Swift An Account of a Battle between the Antient and Modern Books. Hier treten in einer Schlacht antike Philosophen und Dichter gegen moderne Philosophen an. Auf der Seite von Homer, Euklid, Plato, Aristoteles, Herodot und Hippokrates stehen als Alliierte noch der niederländische Theologe Gerardus Vossius der auch eine Abhandlung zur antiken Dichtkunst geschrieben hatte - und der Apologet der Antike, William Temple. Auf der Seite der Modernen stehen hingegen Descartes, Gassendi, Hobbes, aber auch Bacon. ${ }^{12}$ In dieser dichotomisch angelegten Konstellation kommt es nun zu Kampfhandlungen, in denen zum Beispiel ein von Aristoteles abgeschossener Pfeil das eigentliche Ziel, nämlich Bacon verpasst und stattdessen Descartes tödlich in den Kopf trifft. ${ }^{13}$ Die Metapher der Schlacht bei Swift spitzte die von Temple geschaffene direkte Konfrontation von antikem mit modernem Schaffen zu. Was bei Temple die

\footnotetext{
${ }^{10} \mathrm{Vgl}$. Tadié (2013).

${ }^{11}$ Temple, Essay, 457.

${ }^{12}$ Swift, Account, $173 \mathrm{f}$.

${ }^{13}$ Swift, Account, 179. 
Gegenüberstellung als Relativierung der Leistung der Zeitgenossen war, konzipierte Swift als direkte Auseinandersetzung mit dem Ziel, Leistungen und Personen seiner Zeit als unterlegen darzustellen.

Diese Überzeichnung war eine Reaktion auf das Gegenlager, das als Erwiderung auf William Temple lange Traktate über die selbstständige Bedeutung neuer Leistungen in Dichtung, Philosophie, Physik und Naturphilosophie veröffentlichte. Akribisch hatte sich schon 1694, also vier Jahre nach William Temples Veröffentlichung und zehn Jahre vor Jonathan Swift, William Wotton zu Temple geäußert. In Reflections Upon Ancient and Modern Learning, verteidigte der englische Pfarrer, der in seinem breiten Betätigungsfeld auch klassische Literatur übersetzte, die Bedeutung der Modernen. ${ }^{14}$ Er griff Temple direkt an, indem er beispielsweise nach den Möglichkeiten fragte, überhaupt eine Antike zu definieren, wenn doch die Leistungen der Griechen in der Mathematik auf noch älteren und kulturell andersartigen Vorläufern aufbauten. Zudem unterstrich Wotton, dass es in Feldern wie der Naturphilosophie und der Mathematik echte Neuerungen und tatsächlichen Fortschritt geben würde. Gegenüber der antiken Geometrie, beispielsweise, zeigte er, dass in der modernen analytischen Geometrie ganz neue Berechnungsmethoden möglich wurden.

But all this is nothing, in comparison of that boundless extent which the modern mathematicians have carried geometry on to: Which consists in their receiving into it all the curve lines in nature, together with the areas and solids that result from them [...] Add to all this the general methods that have been invented of late for finding the properties of a great number of these curves, for the advancement of opticks, mechanicks, and other parts of philosophy: And let any man of sense give the preference to the ancient geometry if he can. ${ }^{15}$

Diese Verbindung von Geometrie und Algebra, die uns auch im Folgenden noch beschäftigen wird, umfasste auch das Rechnen mit Infinitesimalen, was weit die Möglichkeiten überstieg, die beispielsweise Archimedes zur Verfügung standen. ${ }^{16}$

Es gab, anders als bei Swift, eine ganze Reihe von Autoren eines "dritten Wegs" im ausgehenden siebzehnten und beginnenden 18. Jahrhundert, die eine historischkontextualisierende Argumentationslinie herausstellten, wie Thomas Sprat, Bernard de

\footnotetext{
${ }^{14}$ Vgl. Jones (1961), z.B. 267.

${ }^{15}$ Wotton, Reflections, 162

${ }^{16}$ Wotton, Reflections, 164ff.
} 
Fontenelle, Charles de Saint-Évremond, John Dryden und John Dennis vertreten wurde, in Berufung auf Vorläufer wie Jean Bodin und Francis Bacon. ${ }^{17}$ Obwohl sein Gegenstand besonders die harte Auseinandersetzung im Umfeld der Royal Society ist, zeigt der Literaturwissenschaftler und Wissenschaftshistoriker Richard Foster Jones, dass verschiedene Autoren selbst auf dem Feld der Naturwissenschaften eine große Bandbreite von Meinungen vertraten. Hier fokussierte der Streit hauptsächlich auf die Frage, ob die (aristotelische) Philosophie oder die Experimentalphilosophie à la Francis Bacon wirkliche Erkenntnis bringen könnte. Jones stellt auch die Zwischentöne dar, die eben nicht sich reduzieren lassen auf eine alte Strömung metaphysischen Philosophierens und einer neuen harten Wissenschaftspraxis, obwohl er seine eigene Sympathien für die Bacon'sche Tradition nicht verhehlt. Am Beispiel des englischen Philosophen und Platonikers des 17. Jahrhunderts, Henry More's zeigt Jones, dass ein wissenschaftlich ambitionierter Mann mit seinem wissenschaftlichen Tun eine verbesserte Erklärung von metaphysischen Fragestellungen erreichen wollte. Aus diesem Grund hatte er die Royal Society verlassen und betrieb seine Experimente in Cambridge weiter. ${ }^{18}$

Um die Jahrhundertwende 1700 war es demnach eben keineswegs ausgemacht, dass die Historisierung der Antike sich methodisch und hermeneutisch durchsetzen würde. Vielmehr war die Durchdringung der Gegenwart durch die Antike, also die Unmittelbarkeit beider Epochen häufig noch Gegenstand des Denkens in Literatur und in Wissenschaft.

Hier sind wir wieder bei Isaac Newton und den Principia Mathematica angelangt und damit bei der Frage, welche Rolle die antike Wissenschaft in Newtons Mechanik gespielt hat. An dem anfangs zitierten Verweis auf Pappos lassen sich die verschiedenen Aspekte dieses Einflusses exemplarisch bestimmen. Wir werden zunächst hervorheben, dass in Newtons wissenschaftlichem Werdegang das Studium antiker Autoren grundsätzliche Impulse gab. Zum zweiten wird aus zahlreichen Stellen seiner Publikationen und Korrespondenz offensichtlich, dass Newton sein Projekt einer rationalen Mechanik mittels antiker Vorbilder zu legitimieren suchte-hier ließe sich die transformatorische Fortentwicklung in Newtons Werk erkennen. Schließlich formulierte Newton den Anspruch, dass der Formalismus seiner Mechanik more geometrico antiken Normen von Reinheit und Exaktheit entsprach.

\footnotetext{
${ }^{17}$ Spingarn (1908), cii. Den Schwerpunkt auf William Temple legend, argumentiert Fritz Wagner (1978) ähnlich.

${ }^{18}$ Jones (1961), 250. 
Pappos selbst war ingeniöser Mathematiker, wohl in der ersten Hälfte des vierten

Jahrhunderts nach Christus. Er wirkte in Alexandria, dem Ort des Gelehrtseins der Spätantike par excellence und des Aufeinandertreffens ganz unterschiedlicher Wissenskulturen und mathematischer Strömungen. Pappos’ Mathematische Sammlungen waren Zusammenfassung und Kommentar des spätantiken Wissens. Die erhaltenen Teile wurden durch Federico Commandino 1588 ins Lateinische übersetzt und veröffentlicht. ${ }^{19}$ Der späthellenistische Mathematiker übte einen starken und langanhaltenden Einfluss auf die Geometrie der Renaissance aus. In dieser Tradition sieht Niccolò Guicciardini einen wichtigen Hintergrund für Newtons Auffassung von der Rolle der Geometrie und für die Unterscheidung zwischen synthetischer und analytischer Methode, die für das ganze 17. und 18. Jahrhundert zentral war. $^{20}$

Die Geschichte des Begriffspaares Synthese/Analyse sowohl in der Antike als auch in nachantiken Transformationsprozessen ist äußerst komplex und kann hier nur in den für die Newtonsche Mechanik relevanten Aspekten betrachtet werden. ${ }^{21}$ In der Antike bezeichnete Analyse unter anderem den Schluss vom Unbekannten auf das Bekannte und damit die Rückführung einer zu beweisenden These auf bekannte Sätze. Im siebten Buch der Sammlungen macht Pappos Andeutungen auf eine allgemeine Methode der Analyse zur systematischen Auffindung von Beweisen für geometrische Sätze. Schon in der Renaissance beschäftigten diese Andeutungen die Geometer, die darin den Hinweis auf ein allgemeines Verfahren sahen, das Pappos bekannt war, aber entweder in den verlorengegangen Teilen des Textes enthalten oder von ihm absichtlich verborgen gehalten war. Die Vertreter der neuen Algebra seit François Viète, die die moderne kompakte symbolische Notation in die Mathematik einführten, beriefen sich explizit auf die Tradition einer solchen analytischen Methode. Descartes betonte in seiner Géométrie die Überlegenheit der modernen analytischen Methode, die er am Beispiel eines allgemeinen Beweis eines Problems zeigte, das Pappos nur in einzelnen Fällen geometrisch beweisen konnte. ${ }^{22}$

Newtons erste mathematischen Arbeiten schlossen sich sowohl an die moderne symbolische Algebra von Viète als auch an die cartesische analytische Geometrie an. Auch Newtons Fluxionskalkül, seine bahnbrechende Formulierung der Infinitesimalrechnung, war in diesem

\footnotetext{
${ }^{19}$ Pappos, Mathematicae Collectiones.

${ }^{20}$ Guicciardini (2002).

${ }^{21}$ Otte/Panza (1997) ist eine Sammlung von historischen und philosophischen Arbeiten zur Bedeutung des Begriffspaares in der Geschichte der Mathematik.

${ }^{22}$ Descartes, Discours, 377-387.
} 
formalen Rahmen konzipiert. ${ }^{23}$ Seit den 1670er Jahren nahm Newton unter dem Einfluss seines Lehrers und Vorgängers auf dem Lucasischen Lehrstuhl, Isaac Barrow, eine zunehmend kritische Position in Bezug auf die symbolischen Methoden der modernen Algebra und analytischen Geometrie ein. Guicciardini sieht einen engen Zusammenhang mit Newtons Beschäftigung mit Theologie und biblischer Chronologie seit 1670, in denen Newton ebenfalls die Auffassung vertrat, dass die ursprüngliche und wahre Religion der Menschheit im Laufe der Geschichte zunehmend korrumpiert worden ist. ${ }^{24}$ Auch Descartes wurde von ihm nach 1670, sowohl in seiner Naturphilosophie als auch seiner Mathematik, grundsätzlich kritisiert. In dieser Kritik an Descartes wurde die Gegenüberstellung der Klarheit und Knappheit antiker geometrischer Methoden mit der Obskurität und Langwierigkeit moderner analytischer Methoden zum zentralen Topos. So kritisierte Newton Descartes' Lösung von Pappos' Problem:

To be sure, their [the ancients'] method is more elegant by far than the Cartesian one. For he achieved the result by an algebraic calculus, which, when transposed into words (following the practice of the Ancients in their writings), would prove to be so tedious and entangled as to provoke nausea, nor might it be understood. But they accomplished it by certain simple propositions, judging that nothing written in a different style was worthy to be read, and in consequence concealing the analysis by which they found their constructions. $^{25}$

Newton bewunderte in der Formsprache eines Pappos oder Euklids ein Ideal, das von dem Symbolismus der modernen Algebra nicht erreicht wurde. Denn die mathematische Sprache sollte nicht nur formalen Regeln der Korrektheit folgen, sondern eine Übersetzung von Formeln in Schriftsprache ermöglichen, die sich durch Klarheit und Anschaulichkeit auszeichnen musste. Newton war der Meinung, dass die Alten genau auf diese Weise ihre mathematischen Schriften veröffentlicht hatten ohne die komplexen Wege zu zeigen, auf denen sie zu ihren Erkenntnissen gekommen waren. Diese Übersetzungsfähigkeit fand Newton jedoch nicht in der Notationsmethode René Descartes', dem er vorwarf, unverständlich und obskur zu sein.

Er versuchte, den Fluxionskalkül in eine den Idealen der antiken Geometrie entsprechende Form zu bringen und entwickelte dazu einen Formalismus der „first and last ratios“, das ist

\footnotetext{
${ }^{23}$ Guicciardini (2002), 311-15.

${ }^{24}$ Guicciardini (2002), 316f. Siehe auch Buchwald/Feingold (2013).

${ }^{25}$ Newton, Mathematical Papers, vol. 4, 276 f., zitiert nach Guicciardini (2002), 317. 
ein Verständnis von Differentialquotienten als Grenzwerten von geometrischen Proportionen. Nur in dieser Form traten Differentialquotienten in den Principia auf. Newton berief sich explizit auf die Autorität der Euklidischen Geometrie als Begründung für die Form der Beweise in den Principia Mathematica, in denen er so weit als möglich einen Rückgriff auf analytische Geometrie und Infinitesimalrechnung vermied. Dahingegen kritisierte Newton das Infinitesimalkalkül in der Leibnizschen Form in seinen späteren Jahren heftig als Korruption der klassischen Geometrie. Newton und seine Anhänger behaupteten nicht nur, dass Leibniz die Ideen seiner Infinitesimalrechnung von Newton plagiiert habe, sondern auch, dass seine Darstellung, die auf der expliziten Verwendung unendlich kleiner Größen, der Differentiale, beruhte, widersprüchlich war und zu falschen Resultaten führte. ${ }^{26}$ In dieser Hinsicht wird auch der berühmte Streit über die Priorität in der Entdeckung der Infinitesimalrechnung und über ihre korrekte Formulierung zu einer Fortsetzung der Querelle.

In der eingangs zitierten Vorrede zur ersten Auflage der Principia bezog sich Newton auf Pappos in der Unterscheidung zwischen rationaler Mechanik und praktischer Mechanik und in der Behauptung, dass sie fundamental für die Naturerkenntnis sei. Damit reagierte er auch auf den wissenschaftlichen Diskurs seiner Zeit, auf die untergeordnete Stellung, die angewandte gegenüber reiner Mathematik besaß. Diese recht kurze Allusion an die Antike im Vorwort zur ersten Ausgabe der Principia war tatsächlich nur die Spitze des Eisbergs einer lang andauernden Reflexion Newtons über die Wissenschaft der Antike. Privat äußerte sich Newton ausführlicher zu Plänen, in einer Zweitauflage, das antike Fundament der zeitgenössischen Astronomie, Mathematik und der Physik eingehend darzulegen. Er wolle, so berichtete er seinem Kollegen David Gregory, zeigen, wie sehr die Mathematik und Physik der Principia mit der ursprünglichen Naturphilosophie übereinstimmten, von der in Thales oder Pythagoras nur noch die letzten Reste einer viel älteren Überlieferung überlebten. ${ }^{27}$

By far the greatest changes will be made to Book III. [...] He will show that the most ancient philosophy is in agreement with this hypothesis of his [universal gravitation] as much because the Egyptians and others taught the Copernican system, as he shows from their religion and hieroglyphics and images of the Gods, as because Plato and othersPlutarch and Galileo refer to it-observed the gravitation of all bodies towards all. ${ }^{28}$

\footnotetext{
${ }^{26}$ Hall (2002), 446-447.

${ }^{27}$ McGuire/Rattansi (1966).

${ }^{28}$ Memorandum von David Gregory, 1694. In Turnbull (1961), 384. Quoted after McGuire/Rattansi (1966), 110.
} 
Paolo Casini hat besonders in den Scholien des dritten Buchs, die für eine Überarbeitung der Principia gedacht waren, Newtons oftmals sehr kreativen Umgang mit physikalischen Annahmen antiker Autoren angemerkt. ${ }^{29}$ Casini ist der Meinung, dass Newton das Ziel verfolgte, seine Erkenntnisse - beispielsweise in der Lehre von der Schwerkraft - als Wiederentdeckung antiken Wissens darzustellen. Casinis Arbeit zeigt die Traditionslinien und wissenschaftlichen Übertragungswege auf, denen Newton zu folgen gewillt war. So zitierte der Professor aus Cambridge im Scholium IV über die Schwerkraft des Mondes und seines Orbits neben Plutarch und Diogenes Laertius etwa die Renaissance-Wissenschaftler Natale Conti und Galileo. Sein Abstandsgesetzt begründete Newton demnach mit Diogenes Laertius' Darstellung von Thales, Aristoteles' Darstellung von Pythagoras in der Antike, Macrobius in der Spätantike und Natale Conti in der Renaissance.

Newtons wissenschaftliches Arbeiten, oder zumindest seine Darstellungen, orientierten sich demnach in Teilen an dessen Wissen über antike Mathematik und Mechanik. Darüberhinaus, geradezu alleoleopoetisch, versuchte Netwon, sein Wissen und seine Erkenntnisse als Teile einer antiken Wissenskultur darzustellen.

Unter den zeitgenössischen Mathematikern kann Newtons emphatische Betonung der Autorität der Antike wohl als Extremfall gelten, begründet wohl eher in seinen theologischen und geschichtsphilosophischen Überzeugungen als in mathematischen oder naturphilosophischen. Im Vergleich zu Newton kritisierte beispielsweise Leibniz Euklids axiomatische Methode:

Daher musste Euklid, da er keine distinkt ausgedrückte Idee, d.h. keine Definition der Geraden besaß (denn die, die er vorläufig aufstellt, ist dunkel und hilft ihm bei seinen Beweisen nicht), auf zwei Axiome zurückgreifen, die ihm an Stelle von Definitionen gedient haben und die er in seinen Beweisen verwendet $[\ldots]^{30}$

Im Gegensatz zu Newton sah Leibniz also keineswegs eine unhinterfragbare Autorität in Euklid, er arbeitete vielmehr in seiner Analysis Situs jahrelang an einer Neubegründung der Lehre vom Raum, da er die Euklidische Geometrie nicht für hinreichend hielt.

Die Querelle blieb auch nach dem Tode Newtons im intellektuellen Leben Englands präsent. Im Umfeld der vom Bischof Francis Atterbury geprägten, royalistischen und absolutistischen

\footnotetext{
${ }^{29}$ Casini (1984), 7. auch Morrison (2011), $22 \mathrm{ff}$.

${ }^{30}$ Leibniz, Nouveaux Essais, 487. 
„High Church“ Bewegung in der Anglikanischen Kirche in Christ Church in Oxford schrieben ehemalige Schüler Newtons mathematische Abhandlungen, die das von der Gruppe verfolgte Ideal einer Erhöhung der Antike umsetzen wollten. Die Christ Church Gruppe vertrat den Standpunkt, dass aus antiken Schriften eine höher stehende Moral zu lernen sei. ${ }^{31}$ Mathematiker wie John Keill oder John Colson, oder Mediziner wie John Friend werteten Newton als Zeitgenossen, der diese Weltsicht in seinen Schriften ebenfalls geteilt hatte. Dabei ignorierten sie, wie vielschichtig und widersprüchlich Newtons Leben und Werk war.

1733 veröffentlichte John Keill einen scheinbar ganz aus dem Geist Newtons inspirierten Kommentar zu Euklids Elementen, dem klassischen Werk zur Geometrie. Darin klagte er diejenigen an, die die euklidische Geometrie für obsolet hielten und legte ihr intensives Studium allen Studenten und Schülern ans Herzen. Denn Keill argumentierte, dass entgegen der verbreiteten Annahme eines Fortschritts der Geometrie in letzter Zeit, in den neuen Werken nicht weniger viele Fehler zu finden seien, als dies die Adepten neuer Geometrie den Arbeiten Euklids vorwarfen.

Nay, I dare venture to say, there is not one of these New Systems, wherein there are not more Faults, nay, grosser Paralogisms, than they have been able even to imagine in Euclid. ${ }^{32}$

Ein interessantes Beispiel der Querelle im Bereich der Naturphilosophie ist die Debatte über Newtonische Medizin in England im frühen 18. Jahrhundert. Im Gefolge von Archibald Pitcairne, einem schottischen Arzt und Altertumskundigen, bildete sich eine Gegenbewegung zur cartesischen Medizin, die deren mechanistischen Hypothesen ablehnte und sich auf Newtons Mechanik als Vorbild einer empirischen Wissenschaft berief. Sowohl Pitcairne als auch insbesondere sein Anhänger John Freind, der wiederum Mitglied der Christ Church Gruppe war, sahen in der Newtonischen Medizin eine Rückkehr zu antiken hippokratischen Vorbildern, die sie gegen die „moderne“ Medizin ihrer Gegner verteidigten. Beide waren in ihrer politischen Haltung royalistische Jakobiten, also Verteidiger des Absolutismus gegen den Parlamentarismus. ${ }^{33}$

Andererseits wurde Newton von zahlreichen Philosophen und Naturforschern des 18. Jahrhunderts als Erneuerer der Physik und als Vorreiter der Aufklärung gesehen. Während

\footnotetext{
${ }^{31}$ Friesen (2006), 33-66.

${ }^{32}$ Keill, Geometry, A2 verso.

${ }^{33}$ Friesen (2003).
} 
wir hier auf die Rolle Newtons als intellektuelle Autorität für Vordenker der Aufklärung wie Voltaire $^{34}$ nicht eingehen können, interessiert uns die zweite Gruppe, eine relativ kleine Anzahl von Mathematikern auf dem europäischen Kontinent, die von Newtons Werk ausgehend die Mechanik auf konsequent mathematischer Grundlage neu formulierten. Mit der Transformation der Newtonschen Mechanik zur analytischen Mechanik des 18. Jahrhunderts, die zugleich eine grundsätzliche Transformation seines Werkes bedeutete, befasst sich der letzte Teil dieser Arbeit. Charakteristisch für diese Rezeptionsgeschichte Newtons sind die Übersetzungen und Kommentierungen der Principia, die im frühen 18. Jahrhundert in Frankreich entstanden. In den Jahren 1739-1742 erschien eine ausführlich kommentierte lateinische Ausgabe der Principia, in deren Einleitung es heißt:

Alle, die auch nur den Namen des hochberühmten Autors gehört hatten, wussten, wie geheimnisvoll und zugleich nützlich die Lehren sind, die in den Philosophiae Naturalis Principia Mathematica dargestellt werden. So groß waren die Würde und Erhabenheit des Subjekts, so groß die mehr als geometrische Kürze der Sprache, dass jenes hervorragende Werk für die wenigsten Geometer geschrieben scheint. ${ }^{35}$

Die Ausgabe, häufig - aber unkorrekt - Jesuitenausgabe genannt, wurde besorgt von zwei französischen Franziskanern Thomas Le Seur und François Jacquier unter Beteiligung des Schweizer Gelehrten und Mäzens Jean-Louis Calandrini. ${ }^{36}$ Basierend auf den Arbeiten der Brüder Johann und Jakob Bernoulli, von Pierre de Varignon, und von Leonhard Euler, vollführte diese Edition konsequent die Übersetzung von Newtons Werk in das Infinitesimalkalkül seines Widersachers Leibniz, das sich inzwischen als Standardformulierung der Infinitesimalrechnung etabliert hatte. Es führte damit jene analytischen Methoden wieder in die Principia ein, die Newton so sorgfältig vermieden hatte. Newtons geometrischen Beweisen wurden Satz für Satz Herleitungen im Leibnizschen Infinitesimalkalkül gegenübergestellt, die komplexen diagrammatischen und geometrischen Beweise Newtons durch kompakte analytische Rechnungen ergänzt.

Auch als Émilie du Châtelet in den 1740er Jahren die Principia ins Französische übersetzte, beschränkte sie sich dabei keineswegs auf eine Übersetzung zwischen zwei Sprachen. Aus Latein wird bei Châtelet Französisch; doch obendrein ergänzte die universell gebildete Philosophin, die mit führenden Mathematikern der Académie in Verbindung stand, Newtons

\footnotetext{
${ }^{34}$ Voltaire, Elémens.

${ }^{35}$ Le Seur u. Jacquier, Philosophiae naturalis, I, vii, Z. 1-6.

${ }^{36}$ Bussotti/Pisano (2014). 
euklidische geometrische Formsprache durch Rechnungen im Infinitesimalkalkül. ${ }^{37}$ Diese beiden Ausgaben der Principia repräsentieren die Grundlage der analytischen Mechanik, die Jean d'Alembert, Leonhard Euler und Joseph-Louis Lagrange in der Folgezeit zu einem umfassenden Formalismus entwickeln. Diese Neuformulierung der Mechanik zeigt drei charakteristische Züge:

Die Verwendung neuer mathematischer Mittel, wie zum Beispiel von Differentialgleichungen, des Variationskalküls, und eines abstrakten Funktionsbegriffs. Dieser neue Formalismus wurde durchgängig auf dem Leibnizschen Infinitesimalkalkül aufgebaut, unter weitgehender Missachtung der von Newton nicht zu Unrecht kritisierten Probleme seiner Grundlegung. Diese wurden erst im 19. Jahrhundert ernsthaft adressiert. Das methodologische Ziel einer allgemein anwendbaren Methode zur Lösung mechanischer Probleme, die nicht wie die synthetisch-geometrische von der besonderen Intuition des Geometers abhängt. Ein markantes Beispiel ist das programmatische Vorwort von Lagranges Mechanique analytique, in der er die Vorzüge einer allgemeinen analytischen Methode mit den berühmten Sätzen anpreist: „Man wird in diesem Werke keine Abbildungen finden. Die Methoden, die ich darstelle, erfordern weder Konstruktionen, noch geometrische oder mechanische Betrachtungen, sondern nur algebraische, einem regelmäßigen und gleichförmigen Gange unterworfene Operationen. ${ }^{638}$ Newtons Ideale der Anschaulichkeit und Eleganz sind durch Universalität und Regelmäßigkeit ersetzt.

Die epistemologische Restriktion einer möglichst phänomenologischen Beschreibung mechanischer Systeme, unter Vermeidung Newtons problematischen Kraftbegriffs, der von verschiedenen Seiten als metaphysisch unzulässig und empirisch unbegründet kritisiert wurde. ${ }^{39}$

Diese folgenschwere und fruchtbare Verbindung der Newtonschen Mechanik mit dem Leibnizschen Infinitesimalkalkül, war es, die zur Grundlage der Physik der nächsten 150 Jahre werden sollte und die den Ruhm des englischen Genies prägte. Denn als der uns heute geläufige Ursprung der klassischen Physik konnten Newtons Principia nur deswegen gelten, weil die europäische Rezeption sie in der Formsprache modernisierte.

\footnotetext{
${ }^{37}$ Marquise du Châtelet, Principes.

${ }^{38}$ Lagrange, Mécanique analytique, $\mathrm{i}$.

${ }^{39}$ Auf die Kritik am Newtonschen Kraftbegriff im 18. Jahrhundert kann hier nicht weiter eingegangen werden, sie ist in der Literatur schon ausführlich behandelt worden. Siehe Boudri (2002) für eine neuere Monographie.
} 
Wo blieb in dieser europäischen Rezeption Mitte des 18. Jahrhunderts die Querelle des Anciens et Modernes? Zunächst einmal ist offensichtlich, dass die bei Newton so prominenten Verweise auf die Autorität antiker Wissenschaft in den Texten von d'Alembert, Euler und Lagrange fehlen. Stattdessen beginnt Lagrange seine Mechanique analytique mit einem programmatischen Vorwort, das die innovativen Elemente seiner Mechanik hervorhebt: die Formulierung der Mechanik in der Sprache des analytischen Infinitesimalkalküls, und die Suche nach den allgemeinsten Prinzipien, die der Mechanik zugrunde liegen. Lagrange verzichtet vollkommen auf geometrische Diagramme, und mechanische Probleme werden ausschließlich in Form algebraischer Gleichungen präsentiert. Und die Antike interessiert Lagrange genau nur noch dann, wenn sie Probleme gestellt hat, die in der neuen Physik und Mathematik in einer einfacher zu verstehenden Sprache gelöst wurden. Die Antike ist nicht mehr, wie im eingangs zitierten Vorwort zu Newtons Principia, ein Vorbild in Formsprache und mathematischer Methodik.

In den historischen Bemerkungen von Autoren wie Châtelet, d'Alembert oder Lagrange ist nicht einmal mehr die Frage nach der Überlegenheit der modernen Mechanik aufgeworfen. Stattdessen wird die antike Mechanik eingeordnet in eine nun als selbstverständlich vorausgesetzte Fortschrittsgeschichte der wissenschaftlichen Erkenntnis. Dies ist eines der erstaunlicheren Phänomene der Newton-Rezeption auf dem europäischen Kontinent: Newton selbst wurde zum Kronzeugen eines nicht mehr bezweifelten Fortschrittsparadigmas der europäischen Aufklärung, das an die Stelle seiner Überzeugung von der Korruption des ursprünglichen Wissens der Menschheit tritt. Dieser Wechsel des historischepistemologischen master narratives ist möglicherweise der entscheidendste Paradigmenwechsel im Übergang von der Wissenschaft der Spätrenaissance zu der der Aufklärung. Es ist hier jedoch nicht der Platz, für eine so allgemeine These zu argumentieren, unser Interesse gilt der fundamentalen Veränderung der Rolle der antiken Wissenschaft für den zeitgenössischen Diskurs.

Die antike Mechanik war für die Renaissance eine Auctoritas, die, ob sie als Begründung eigener Argumente oder als Angriffspunkt für Kritik genützt wurde, immer zugleich als zeitgenössisches Wissen verstanden wurde. Sie stand für das von ihr Bezeichnete genauso wie es das experimentelle Phänomen oder der theoretische Begriff taten. Die archimedische Maschine ist die Maschine der Baustelle, des Bergwerks oder der Werkstatt. Wie Joyce van Leeuwen in ihrem Beitrag detailliert belegt, entspricht dem eine allelopoietische Konstruktion antiker Wissenschaft, die, wenn sie nur von den Verfälschungen der Überlieferung befreit ist, 
direkt die Probleme der zeitgenössischen Praxis löst. Newton treibt diese Tradition auf die Spitze: genauso wie er überzeugt war, dass die wahre Religion durch Jahrtausende der Verfälschung bis zur Unkenntlichkeit verstümmelt worden war, war auch die wahre Wissenschaft des Altertums fast gänzlich vor der Nachwelt verborgen. Sie musste genauso aus verstreuten Hinweisen durch akribische Schlussfolgerungen rekonstruiert werden wie die wahre Religion. So entstand ein Antikenbild, das einerseits die Andersartigkeit der Antike betonte, aber auch radikal von unserem verschieden war.

Nichts mehr von diesem Glauben an die Überlegenheit der Antike findet sich in den historischen Abrissen von d'Alembert oder Lagrange. Die Reste der antiken Mechanik finden sich nur mehr als isolierte Lehrsätze, die zwar historisch verortet werden wie die Hebelgesetze in der Zuschreibung an Archimedes, jedoch stets von der Frage begleitet, wie sie sich allgemeiner begründen lassen als von den antiken Autoren. So beschreibt Lagrange die Rezeption von Archimedes' Begründung des Hebelgesetzes bei Stevin, Galileo und Huyghens nahezu ausschließlich unter dem Gesichtspunkt, wie diese Autoren den archimedischen Beweis zu verbessern versucht haben, und kritisiert diese Verbesserungsversuche. Diese Rezeptionsgeschichte kann Lagrange nun für sein Anliegen nutzen, seine Erklärung und mathematische Darstellung als verbesserte Lösung zu präsentieren. ${ }^{40}$ Was bleibt vom traditionellen Wissen der Mechanik wird in einer neuen Sprache, dem analytischen Infinitesimalkalkül formuliert und in einen völlig neuen Begründungszusammenhang, der Suche nach einheitlichen Fundamentalprinzipien, eingeordnet. Diese Transformation ist in der Terminologie von Bergemann et al. (2011) also sowohl eine Disjunktion, eine Einkleidung des traditionellen empirischen Gehaltes der praktischen Mechanik in einen neuen Formalismus, als auch eine Umdeutung, die Neuinterpretation traditioneller physikalischer Grundbegriffe im Zusammenhang eines mathematischen Theoriengebäudes. Beide Transformationsformen sind für Lagrange wesentlich miteinander verbunden, da für ihn die Einheit des analytischen Formalismus eine notwendige Voraussetzung für die Einheit der analytischen Methode als Lösungsverfahren mechanischer Probleme ist.

Dadurch entsteht erstmals eine historische Distanz zur antiken Wissenschaft, die nicht mehr einfach Wissenschaft per se ist, sondern ein historisches Phänomen und vom modernen Wissen über die Natur klar geschieden. Genauso wie die klassische Physik erst in der Reflexion über die Relativitäts- und die Quantentheorie entstand, ist die antike Mechanik ein

\footnotetext{
${ }^{40}$ Lagrange, Mécanique analytique. 4.
} 
Produkt der Formalisierung und Kanonisierung der Newtonschen Mechanik. Auch dies ist ein Prozess der Allelopoiese, der zumindest die Grundlage dafür legt, die antike Wissenschaft als historisches Kulturphänomen überhaupt wahrzunehmen. Man kann hier durchaus eine Parallele erkennen zur Entstehung des Bewusstseins von der Eigenart antiker Kunst durch den Beginn wissenschaftlicher Ausgrabungskampagnen im 18. Jahrhundert. Andererseits wird die Naturwissenschaft im 18. Jahrhundert noch nicht, wie andere antike Kulturphänomene, selbst zum Objekt einer wissenschaftlichen Geschichtsschreibung. Dadurch unterscheidet sich der Ausgang der Querelle in der Mechanik von dem in der Literatur- und Altertumswissenschaft. Die erste konsequente historische Beschreibung der Naturwissenschaft wird erst im folgenden Jahrhundert William Whewell geben. Bis dahin bleibt das Interesse an antiker Wissenschaft weitgehend reduziert auf eine „Lehrbuchhistoriographie“, die sich darauf beschränkt, einzelne Phänomene oder Theoreme bestimmten Entdeckern zuzuschreiben, wie dies in Lagranges gerade besprochener Einleitung zur Analytischen Mechanik oder auch in d'Alemberts Discours preliminaire zur Encyclopédie geschieht. Während also die Wissensbestände der vorklassischen und antiken Mechanik in einer transformierten Form in der analytischen Mechanik fortexistieren, verlieren sie in dieser transformierten Form gerade ihre historische und lokale Charakteristik. Die antike Wissenschaft ist in eine universelle Fortschrittsgeschichte eingebettet, in der die Antike vom Vorbild zum Vorläufer wird.

\section{QUELLENTEXTE}

Châtelet, Gabrielle-Émilie Le Tonnelier Bréteuil, Marquise du, Principes mathématiques de la philosophie naturelle, Vol. 1. Paris 1759.

Descartes, René, Discours de la méthode pour bien conduire sa raison \& chercher la verité dans les sciences : plus la diotrique, les meteores, et la geometrie, qui sont des essais de cete méthode (1637), in: Oeuvres de Descartes, Vol. 6, hg. v. Charles Adam/Paul Tannery, Paris, 1982.

Keill, John, Euclid's Elements of Geometry, London 1733.

Lagrange, Joseph Louis, Mécanique Analytique, Paris 1788.

Le Seur, Thomas und François Jacquier, Philosophiae naturalis principia mathematica, Genf 1739.

Leibniz, Gottfried Wilhelm, Nouveaux essais sur l'entendement humain, in: Neue Abhandlungen über den menschlichen Verstand, übs. v. Ernst Cassirer, Hamburg, Neuausgabe 1996. 
Newton, Isaac, The Mathematical Papers of Isaac Newton, 8 vols., hg. von D. Thomas Whiteside, Cambridge 1967-81.

Newton, Isaac, The Principia: Mathematical Principles of Natural Philosophy (1687), übs. und hg. v.

I. Bernard Cohen und Anne Whitman, Berkeley/Los Angeles/London 1999.

Pappus Alexandrinus, Pappi Alexandri Mathematicae Collectiones, übs. und hg. v. Federico Commandino, Bologna 1588.

Swift, Jonathan, Tale of a Tub. Written for the Universal Improvement of Mankind. To which is added, an account of a battle between the antient and modern books in St. James's library, London 1751.

Temple, William, „Essay upon ancient and modern learning (1690)“ in: The Works of Sir William Temple, vol. III. London, printed for F.C. and J. Rivington et.al (1814), S. 444-486.

Voltaire, Elémens de la philosophie de Neuton: mis à la portée de tout le monde, Amsterdam 1738.

Wotton, William, Reflections upon Ancient and Modern Learning, London 1694.

\section{FORSCHUNGSLITERATUR}

Bergemann, Lutz et al., „Transformation: Ein Konzept zur Erforschung kulturellen Wandels“, in: Transformation: Ein Konzept zur Erforschung kulturellen Wandels, hg. v. Hartmut Böhme et al., München 2011, 39-56.

Buchwald, Jed Z./Feingold, Mordechai, Newton and the Origin of Civilization, Princeton 2013.

Bussotti, Paolo/ Pisano, Raffaele, „On the Jesuit Edition of Newton's Principia. Science and Advanced Researches in the Western Civilization“, in: Scientific Research 3 (1) (2014), 33-55.

Boudri, J. Christiaan, What was Mechanical about Mechanics: The Concept of Force between Metaphysics and Mechanics from Newton to Lagrange, Dordrecht/Boston/London 2002.

Casini, Paolo, „Newton: The Classical Scholia“, in: History of Science 22 (1984), 1-58.

Cohen, I. Bernard, The Newtonian Revolution. With Illustrations of the Transformation of Scientific Ideas, London/New York 1980.

Cohen, I. Bernard/Smith, George E., The Cambridge Companion to Newton, Cambridge 2002.

Duval, Paul-Marie, „La notion de Gaulois: une longue confusion“, in: Travaux sur la Gaule: 1946-1986 : textes revus et mis à jour, hg. v. Paul-Marie Duval, François-Charles Uginet, Marcelle Hartmann, Rom 1989, 177-185.

Friesen, John, „Archibald Pitcairne, David Gregory and the Scottish Origins of English Tory Newtonianism, 1688-1715“, History of Science 41 (2003): 163-91. 
Friesen, John, „Hutchinsonianism and the Newtonian Enlightenment“, Centaurus 48 (2006): 40-49.

Guicciardini, Niccolò, Reading the Principia: The Debate on Newton's Mathematical Methods for Natural Philosophy from 1687 to 1736, Cambridge 1999.

Guicciardini, Niccolò, „Analysis and Synthesis in Newton's Mathematical Work“, in: The Cambridge Companion to Newton, hg. v. I. Bernard Cohen, George E. Smith, Cambridge 2002, 308328.

Hall, A. Rupert, „Newton versus Leibniz: from Geometry to Metaphysics“ in: hg. v. I. Bernard Cohen, George E. Smith, Cambridge 2002, 431-454.

Hardt, Dietrich, „Über die Geburt der Antike aus dem Geist der Moderne“, in: International Journal of the Classical Tradition 1 (1) (1994), 89-106.

Jones, R. F., Ancients and Moderns. A Study of the Rise of Scientific Movement in SeventeenthCentury England, St. Louis 1961.

Levine, Joseph M., „Ancients and Moderns Reconsidered“, in: Eighteenth-Century Studies 15 (1) (1981), 72-89.

McGuire, James E./ Rattansi, Piyo M., „Newton and the ,Pipes of Pan'“, in: Notes and Records of the Royal Society of London 21 (2) (1966), 108-143.

Nicolet, Claude, „Des Belles-Lettres à l'érudition: l'Antiquité gréco-romaine à l'Académie au XVIIIe siècle“, in: Comptes rendus des séances de l'Académie des Inscriptions et Belles-Lettres 145 (4) (2001), 1627-1637.

Otte, Michael/Panza, Marco, Analysis and Synthesis in Mathematics: History and Philosophy, Dordrecht/Boston/London 1997.

Rigault, Hyppolyte, Histoire de la Querelle des Anciens et Modernes (1856), New York 1963.

Spingarn, Joel Elias, Critical Essays of the Seventeenth Century (1605-1650), tome 1, Oxford 1908.

Tadié, Alexis, „Peut-on traduire les querelles? De la Querelle des anciens et des modernes à la Battle of the Books", in: Littératures classiques 81 (2013), 211-226.

Thoma, Heinz, „Die ästhetischen Theorien im Überblick. Die ,querelle des anciens et des modernes"“, in: Grundriss der Geschichte der Philosophie. Die Philosophie des 18. Jahrhunderts, hg. v. H. Holzhey, J. Rohbeck and F. Ueberweg, Basel 2008 (=Frankreich, Bd. 2), 757-766.

Turnbull, H. W. (Herausgeber), The Correspondence of Isaac Newton, Vol. 3, Cambrige 1961.

Wagner, Fritz, „Entstehen der Geschichte als Wissenschaft“", in: Berichte zur Wissenschaftsgeschichte 1 (1978), 43-50.

Warnick, Barbara, „The Old Rhetoric Vs. the New Rhetoric. The Quarrel Between the Ancients and the Moderns", in: Communication Monographs 49 (1982), 263-276. 
Whewell, William, History of the Inductive Sciences: From the Earliest to the Present Time, London 1837. 


\section{MAX-PLANCK-INSTITUT FÜR WISSENSCHAFTSGESCHICHTE}

\section{Max Planck Institute for the History of Science}

Preprints since 2013 (a full list can be found at our website)

437 Jürgen Renn Schrödinger and the Genesis of Wave Mechanics

438 Pietro Daniel Omodeo L'iter europeo del matematico e medico scozzese Duncan Liddel

439 Irina Tupikova \& Klaus Geus The Circumference of the Earth and Ptolemy's World Map

440 Pietro Daniel Omodeo und Jürgen Renn Das Prinzip Kontingenz in der Naturwissenschaft der Renaissance

441 Horst Kant und Jürgen Renn Eine utopische Episode - Carl Friedrich von Weizsäcker in den Netzwerken der Max-Planck-Gesellschaft

442 William G. Boltz and Matthias Schemmel The Language of 'Knowledge' and 'Space' in the Later Mohist Canon (TOPOI - Towards a Historical Epistemology of Space)

443 Stefano Bordoni Looking for a Rational Thermodynamics in the late XIX century

444 Sonja Brentjes and Jürgen Renn The Arabic Transmission of Knowledge on the Balance

445 Horst Nowacki Archimedes and Ship Design

446 Matthias Schemmel Elements of a Historical Epistemology of Space (TOPOI - Towards a Historical Epistemology of Space]

447 Martin Thiering and Wulf Schiefenhövel Spatial Concepts in Non-Literate Societies: Language and Practice in Eipo and Dene Chipewyan (TOPOI - Towards a Historical Epistemology of Space]

448 Jürgen Renn Einstein as a Missionary of Science

449 Hubert Laitko Der Ambivalenzbegriff in Carl Friedrich von Weizsäckers Starnberger Institutskonzept

450 Stefano Bordoni When Historiography met Epistemology. Duhem's early philosophy of science in context

451 Renate Wahsner Tausch - Allgemeines - Ontologie oder Das Auseinanderlegen des Konkreten und seine Aufhebung

452 Jens Høyrup Algebra in Cuneiform. Introduction to an Old Babylonian Geometrical Technique

453 Horst Nowacki Zur Vorgeschichte des Schiffbauversuchswesens

454 Klaus Geus and Mark Geller (eds.) Esoteric Knowledge in Antiquity (TOPOI - Dahlem Seminar for the History of Ancient Sciences Vol. II]

455 Carola Sachse Grundlagenforschung. Zur Historisierung eines wissenschaftspolitischen Ordnungsprinzips am Beispiel der Max-Planck-Gesellschaft (1945-1970)

456 David E. Rowe and Robert Schulmann General Relativity in the Context of Weimar Culture

457 F. Jamil Ragep From Tūn to Turun: The Twists and Turns of the Ṭūsi-Couple

458 Pietro Daniel Omodeo Efemeridi e critica all'astrologia tra filosofia naturale ed etica: La contesa tra Benedetti e Altavilla nel tardo Rinascimento torinese

459 Simone Mammola Il problema della grandezza della terra e dell'acqua negli scritti di Alessandro Piccolomini, Antonio Berga e G. B. Benedetti e la progressiva dissoluzione della cosmologia delle sfere elementari nel secondo '500 
460 Stefano Bordoni Unexpected Convergence between Science and Philosophy: A debate on determinism in France around 1880

461 Angelo Baracca Subalternity vs. Hegemony - Cuba's Unique Way of Overcoming Subalternity through the Development of Science

462 Eric Hounshell \& Daniel Midena "Historicizing Big Data" Conference, MPIWG, October 31 - November 2, 2013 Report

463 Dieter Suisky Emilie Du Châtelet und Leonhard Euler über die Rolle von Hypothesen. Zur nach-Newtonschen Entwicklung der Methodologie

464 Irina Tupikova Ptolemy's Circumference of the Earth (TOPOI - Towards a Historical Epistemology of Space]

465 Irina Tupikova, Matthias Schemmel, Klaus Geus Travelling along the Silk Road: A new interpretation of Ptolemy's coordinates

466 Fernando Vidal and Nélia Dias The Endangerment Sensibility

467 Carl H. Meyer \& Günter Schwarz The Theory of Nuclear Explosives That Heisenberg Did not Present to the German Military

468 William G. Boltz and Matthias Schemmel Theoretical Reflections on Elementary Actions and Instrumental Practices: The Example of the Mohist Canon (TOPOI - Towards a Historical Epistemology of Space]

469 Dominic Olariu The Misfortune of Philippus de Lignamine's Herbal or New Research Perspectives in Herbal Illustrations From an Iconological Point of View

470 Fidel Castro Díaz-Balart On the Development of Nuclear Physics in Cuba

471 Manfred D. Laubichler and Jürgen Renn Extended Evolution

472 John R. R. Christie Chemistry through the 'Two Revolutions': Chemical Glasgow and its Chemical Entrepreneurs, 1760-1860 
